\title{
Exame citológico em medicina veterinária: estudo retrospectivo de 11.468 casos $(1994-2008)^{1}$
}

\author{
Rodolfo F.A. Ventura², Marcia M. Colodel ${ }^{2 *}$ e Noeme S. Rocha²
}

\begin{abstract}
Ventura R.F.A., Colodel M.M. \& Rocha N.S. 2012. [Cytological examination in veterinary medicine: Retrospective study of 11,468 cases (1994-2008).] Exame citológico em medicina veterinária: estudo retrospectivo de 11.468 casos (1994-2008). Pesquisa Veterinária Brasileira 32(11):1169-1173. Departamento de Clínica Veterinária, Faculdade de Medicina Veterinária, Universidade Estadual Paulista, Cx. Postal 585, Distrito de Rubião Júnior s/n, Botucatu, SP 18618-970, Brazil. E-mail: mmc@fmvz.unesp.br

A retrospective study was conducted over the results of cytological examination in the files of the Department of Cytopathology, Laboratory of Animal Pathology, School of Veterinary Medicine and Animal Science, São Paulo State University, Botucatu, SP, Brazil, from January 1994 to December 2008. Out of 139,986 animals referred to the teaching Veterinary Hospital, 11,468 (8.2\%) were submitted to cytological examination. From these animals, $57.28 \%$ had a neoplastic lesion, $19.28 \%$ had inflammatory lesions, and $14.79 \%$ had non-neoplastic processes (immune-mediated, degenerative, hyperplastic, or dysplastic condition). By means of cytology, $7.28 \%$ of the cases were unable to be diagnosed, and there was no recorded diagnostic information in $1.37 \%$ of cases. The species that more commonly had samples submitted to cytology was the dog (92.06\%), followed by cats $(4.08 \%)$, cattle $(1.77 \%)$, and horses $(1.30 \%)$. As to the predominance of the observed processes, greater importance was given to neoplastic lesions. These were prevalent in dogs (59.18\%), with a mean age of 119.60 months, and affecting mainly females (61.61\%). The annual increase observed over the 15 years of application of cytology demonstrates that the cytological examination is a method of great importance to confirm, suggest, or exclude the diagnosis of various diseases, including cancer, in all animal species.
\end{abstract}

INDEX TERMS: Cytology, epidemiology, prevalence, animals.

RESUMO.- Foi realizado um estudo retrospectivo dos resultados dos exames citológicos provenientes dos arquivos do Serviço de Citopatologia do Laboratório de Patologia Animal, Faculdade de Medicina Veterinária e Zootecnia, Universidade Estadual Paulista, Botucatu, São Paulo, entre janeiro de 1994 e dezembro de 2008. Do total de 139.986 animais atendidos no Hospital Veterinário da instituição, $11.468(8,2 \%)$ foram encaminhados para exame citológi-

\footnotetext{
${ }^{1}$ Recebido em 22 de fevereiro de 2012.

Aceito para publicação em 1 de setembro de 2012.

${ }^{2}$ Departamento de Clínica Veterinária, Faculdade de Medicina Veterinária (FMVZ), Universidade Estadual Paulista (Unesp), Cx. Postal. 585, Distrito de Rubião Júnior s/n, Botucatu, SP 18618-970, Brasil. *Autor para correspondência: mmc@fmvz.unesp.br. Outros e-mails de autores: franksson_vet@hotmail.com; rochanoeme@fmvz.unesp.br
}

co. Desses diagnósticos, $57,28 \%$ corresponderam a lesões neoplásicas, $19,28 \%$ a lesões inflamatórias e $14,79 \%$ a processos não neoplásicos (imunológicos, degenerativos, hiperplásicos ou displásicos). Não foi possível concluir o diagnóstico por meio do exame citológico em 7,28\% dos casos e não haviam informações diagnósticas registradas em $1,37 \%$ dos casos. Dentre os animais que mais receberam indicação para exame citológico destacaram-se os caninos $(92,06 \%)$, seguidos pelos felinos $(4,08 \%)$, bovinos $(1,77 \%)$ e equinos $(1,30 \%)$. Lesoes neoplásicas foram as mais comumente observadas e foram mais prevalentes nos cães $(59,18 \%)$, afetando principalmente animais com idade média de 119,60 meses e principalmente fêmeas (61,61\%). 0 incremento anual observado ao longo dos 15 anos de aplicação da citologia demonstra que o exame cito- 
lógico é um método de suma importância para confirmar, sugerir ou afastar o diagnóstico de diversas afecções, inclusive neoplasia, em todas as espécies animais.

TERMOS DE INDEXAÇÃO: Citologia, epidemiologia, prevalência, animais.

\section{INTRODUÇÃO}

Nas últimas décadas, a busca de soluções relativas aos problemas clínicos pertinentes às diferentes espécies animais tem demandado esforços no sentido de desenvolver novos e mais elaborados métodos de diagnóstico. No entanto, quando os recursos para a utilização de exames sofisticados são reduzidos, a utilização de métodos ainda precisos, mas de baixa complexidade e custo deve ser considerada. A citologia, dentro desse contexto, é um exame de grande valor diagnóstico, pois os resultados obtidos com o uso adequado da técnica podem ser muito importantes tanto para a escolha do tratamento, quanto para evitar uma intervenção cirúrgica arriscada e desnecessária (Meyer 2001, Simon et al. 2009).

Além disso, o uso da citologia como meio de diagnóstico em medicina veterinária possui as vantagens de ter baixo custo, ser minimamente invasivo, apresentar segura execução, permitir ampla superfície de amostragem e o curto tempo entre o processo de diagnóstico e o resultado são outras vantagens desse exame (Magalhães et al. 2001, Simeonova et al. 2004, Teixeira et al. 2010). No entanto, resultados clinicamente relevantes dependem, dentre outros fatores, da obtenção de boas amostras que contenham áreas representativas da lesão, que não contenham artefatos ou contaminação, e que sejam coradas por métodos que evidenciem tanto critérios citoplasmáticos quanto critérios nucleares (Allen et al. 1986, Ménard et al. 1986, Larkin 1994, Raskin $\&$ Meyer 2009). Adicionalmente, um exame bem sucedido depende também da interpretação combinada dos achados citológicos, clínicos e de imagem (Meyer 2001).

O objetivo do presente estudo retrospectivo foi avaliar os aspectos epidemiológicos e determinar a prevalência do exame citológico de afecções que acometeram animais atendidos no Serviço de Citopatologia do Laboratório de Patologia Veterinária da Faculdade de Medicina Veterinária e Zootecnia (FMVZ), Universidade Estadual Paulista
(Unesp), Botucatu, São Paulo, durante 15 anos de aplicabilidade do método.

\section{CITOLOGIA NA FMVZ, UNESP-BOTUCATU, SÃO PAULO}

Entre janeiro de 1994 e dezembro de 2008 foi realizado um levantamento retrospectivo dos resultados dos exames citológicos provenientes dos arquivos do Serviço de Citopatologia do Laboratório de Patologia Veterinária, FMVZ, Unesp-Botucatu/SP. Foram considerados os diagnósticos citológicos relacionados tanto às estruturas externas quanto aos órgãos internos dos animais atendidos.

Os resultados dos exames citológicos de cada caso foram avaliados e com base na afecção principal, as lesões foram classificadas em processo inflamatório e não-inflamatório. Os processos não-inflamatórios foram subclassificados em não-neoplásico e neoplásico. Consideraram-se como não-neoplásico os casos com base em disturbios imunológicos, degenerativos, hiperplásicos e displásicos. Casos em que o diagnóstico não pode ser concluido com base nos achados citológicos ou em amostras insuficientes foram considerados inconclusivos e casos onde não haviam informações registradas dos achados citológicos foram considerados como sem informações.

$\mathrm{Na}$ análise epidemiológica, foi estabelecida a prevalência quanto à classificação citológica, ano de atendimento, espécie animal, idade em meses e sexo. Em relação ao sexo, os animais foram classificados como macho e fêmea, independente de serem castrados ou não. Também foi estabelecida a prevalência dos casos encaminhados para citologia em relação ao total de registros de casos clínicos atendidos no Hospital Veterinário (HV) da FMVZ, Unesp-Botucatu/SP, durante o período de tempo estudado.

Uma vez concluída a coleta de dados junto aos arquivos, procedeu-se a contagem e tabulação dos resultados, sendo considerados os números absolutos e percentuais dos grupos de lesões sobre o total geral e individualmente em cada grupo.

\section{Casuística observada durante os $\mathbf{1 5}$ anos de estudo}

Entre janeiro de 1994 e dezembro de 2008 foram atendidos, no HV da FMVZ, Unesp-Botucatu/SP, 139.986

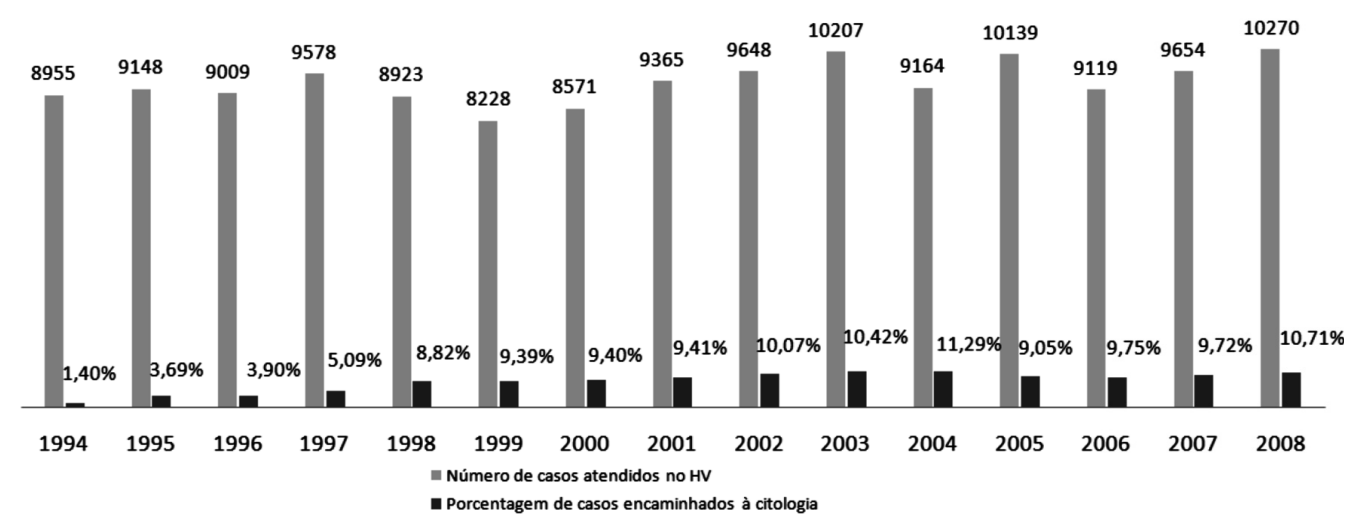

Fig.1. Porcentagem de animais encaminhados para exame citológico em relação ao total de casos atendidos no Hospital Veterinário (HV) da FMVZ-Unesp, Botucatu/SP, de janeiro de 1994 a dezembro de 2008. 
animais. Desses, 11.468 (8,2\%) tiveram amostras encaminhadas para exame citológico. Constatou-se um aumento gradual no percentual de amostras encaminhadas para exame citológico em relação ao total de casos atendidos no HV entre 1994 e 2004. Em 2005 observou-se ligeira queda de $2,24 \%$ na submissão de amostras, com novo incremento nos anos subsequentes (Fig.1).

A distribuição dos diferentes tipos de afecções classificadas pelo exame citológico em relação ao ano está representada no Quadro 1. Dos 11.468 exames citológicos realizados, $6.569(57,28 \%)$ corresponderam a lesões neoplásicas, $2.211(19,28 \%)$ a lesões inflamatórias e 1.696 $(14,79 \%)$ a processos não-neoplásicos. Diagnósticos inconclusivos perfizeram um total de 835 casos $(7,28 \%)$ e não havia informações diagnósticas registradas em 157 casos $(1,37 \%)$.

A maior ocorrência de lesões neoplásicas em relação às demais alterações foi observada ao longo dos 15 anos avaliados. As lesões inflamatórias ocuparam o segundo lugar em ocorrência no período de 1994-2004, no entanto, a partir de 2005, houve um incremento das lesões não- neoplásicas e estas passaram a ocupar o segundo lugar (Quadro 1).

A distribuição quanto às espécies animais afetadas pelos diferentes tipos de lesões está representada no Quadro 2. Dentre os animais que mais receberam indicação para exame citológico destacaram-se os 10.558 caninos $(92,06 \%)$, seguido por 468 felinos (4,08\%), 203 bovinos $(1,77 \%)$ e 149 equinos $(1,30 \%)$. Nos caninos, ocorreram com maior

Quadro 1. Classificação citológica das afecções acometendo animais atendidos no

Serviço de Citopatologia do Laboratório de Patologia Veterinária, FMVZ-Unesp, Botucatu/SP, de janeiro de 1994 a dezembro de 2008

\begin{tabular}{ccccccc}
\hline Ano & \multicolumn{5}{c}{ Classificação citológica } & Total \\
\cline { 2 - 5 } & $\begin{array}{c}\text { Inflamatórias } \\
\mathrm{n}^{*}(\%)\end{array}$ & $\begin{array}{c}\text { Não-inflamatórias } \\
\mathrm{n}^{*}(\%)\end{array}$ & $\begin{array}{c}\text { Inconclusivas } \\
\mathrm{n}^{*}(\%)\end{array}$ & $\begin{array}{c}\text { Sem informação } \\
\mathrm{n}^{*}(\%)\end{array}$ \\
\cline { 3 - 5 } & & Não-neoplásicas & Neoplásicas & & & \\
\hline 1994 & $26(20,64)$ & $14(11,11)$ & $53(42,06)$ & $25(19,84)$ & $08(6,35)$ & $126(1,10)$ \\
1995 & $84(24,85)$ & $20(5,92)$ & $180(53,25)$ & $47(13,91)$ & $07(2,07)$ & $338(2,95)$ \\
1996 & $100(28,41)$ & $44(12,50)$ & $187(53,12)$ & $17(4,83)$ & $04(1,14)$ & $352(3,07)$ \\
1997 & $101(20,70)$ & $54(11,06)$ & $321(65,78)$ & $12(2,46)$ & $00(0,00)$ & $488(4,25)$ \\
1998 & $151(19,19)$ & $84(10,67)$ & $463(58,83)$ & $88(11,18)$ & $01(0,13)$ & $787(6,86)$ \\
1999 & $251(32,47)$ & $105(13,58)$ & $379(49,03)$ & $38(4,92)$ & $00(0,00)$ & $773(6,74)$ \\
2000 & $229(28,41)$ & $97(12,03)$ & $430(53,35)$ & $49(6,08)$ & $01(0,13)$ & $806(7,02)$ \\
2001 & $208(23,61)$ & $130(14,75)$ & $464(52,67)$ & $77(8,74)$ & $02(0,23)$ & $881(7,68)$ \\
2002 & $185(19,03)$ & $139(14,30)$ & $518(53,29)$ & $124(12,76)$ & $06(0,62)$ & $972(8,47)$ \\
2003 & $196(18,42)$ & $121(11,38)$ & $653(61,37)$ & $94(8,83)$ & $00(0,00)$ & $1064(9,28)$ \\
2004 & $163(15,75)$ & $146(14,11)$ & $656(63,38)$ & $65(6,28)$ & $05(0,48)$ & $1035(9,02)$ \\
2005 & $115(12,51)$ & $151(16,43)$ & $593(64,53)$ & $49(5,33)$ & $11(1,20)$ & $919(8,01)$ \\
2006 & $106(11,92)$ & $245(27,56)$ & $472(53,09)$ & $32(3,60)$ & $34(3,83)$ & $889(7,75)$ \\
2007 & $114(12,16)$ & $141(15,03)$ & $613(65,35)$ & $59(6,29)$ & $11(1,17)$ & $938(8,18)$ \\
2008 & $182(16,55)$ & $205(18,64)$ & $587(53,36)$ & $59(5,36)$ & $67(6,09)$ & $1100(9,60)$ \\
Total & $2211(19,28)$ & $1696(14,79)$ & $6569(57,28)$ & $835(7,28)$ & $157(1,37)$ & $11468(100)$
\end{tabular}

$\overline{* n}=$ número de animais.

Quadro 2. Distribuição quanto à espécie e sexo animal em relação à classificação citológica das afecções acometendo animais atendidos no Serviço de Citopatologia do Laboratório de Patologia Veterinária, FMVZ-Unesp, Botucatu/SP, de janeiro de 1994 a dezembro de 2008

\begin{tabular}{|c|c|c|c|c|c|c|c|c|c|c|c|}
\hline \multirow[t]{3}{*}{ Espécie } & \multirow{3}{*}{\multicolumn{2}{|c|}{$\begin{array}{c}\text { Inflamatórias } \\
\frac{\mathrm{n}^{*}}{\text { Macho Fêmea }}\end{array}$}} & \multicolumn{2}{|c|}{$\begin{array}{c}\text { Não-inflamatórias } \\
\mathrm{n}^{*}\end{array}$} & \multicolumn{4}{|c|}{$\begin{array}{c}\text { Inconclusivas } \\
\mathrm{n}^{*} \\
\end{array}$} & \multicolumn{2}{|c|}{$\begin{array}{c}\text { Sem informação } \\
n^{*} \\
\end{array}$} & \multirow[t]{3}{*}{ Total } \\
\hline & & & \multirow[t]{2}{*}{ Macho } & \multirow[t]{2}{*}{ Fêmea } & \multicolumn{2}{|c|}{ Não-neoplásicas } & \multicolumn{2}{|c|}{ Neoplásicas } & \multirow[t]{2}{*}{ Macho } & \multirow[t]{2}{*}{ Fêmea } & \\
\hline & & & & & Macho & Fêmea & Macho & Fêmea & & & \\
\hline Avícola & 04 & 04 & 03 & 06 & 02 & 05 & 01 & 01 & 00 & 00 & 26 \\
\hline Bovina & 17 & 17 & 14 & 13 & 12 & 19 & 108 & 02 & 01 & 00 & 203 \\
\hline Canina & 991 & 959 & 748 & 785 & 2382 & 3866 & 294 & 381 & 79 & 73 & 10558 \\
\hline Caprina & 01 & 02 & 01 & 02 & 00 & 02 & 00 & 01 & 00 & 00 & 9 \\
\hline Equina & 39 & 19 & 09 & 09 & 37 & 18 & 09 & 07 & 02 & 00 & 149 \\
\hline Felina & 65 & 69 & 43 & 50 & 87 & 125 & 14 & 13 & 01 & 01 & 468 \\
\hline Leporina & 05 & 01 & 00 & 03 & 00 & 03 & 00 & 00 & 00 & 00 & 12 \\
\hline Mustela & 02 & 00 & 01 & 00 & 00 & 00 & 00 & 00 & 00 & 00 & 3 \\
\hline Ovina & 04 & 06 & 04 & 02 & 00 & 02 & 00 & 00 & 00 & 00 & 18 \\
\hline Primata & 00 & 01 & 00 & 00 & 00 & 01 & 00 & 00 & 00 & 00 & 2 \\
\hline Roedora & 03 & 01 & 02 & 01 & 02 & 06 & 01 & 01 & 00 & 00 & 17 \\
\hline Réptil & 00 & 01 & 00 & 00 & 00 & 00 & 00 & 01 & 00 & 00 & 2 \\
\hline Suína & 00 & 00 & 00 & 00 & 00 & 00 & 00 & 01 & 00 & 00 & 1 \\
\hline Total & 1131 & 1080 & 825 & 871 & 2522 & 4047 & 427 & 408 & 83 & 74 & 11468 \\
\hline
\end{tabular}

*n = número de animais. 
frequência as afecções neoplásicas (6.248 casos; 59,18\%) e na sequência vieram os processos inflamatórios $(1.950$ casos; $18,47 \%$ ) e não-neoplásicos ( 1.533 casos; $14,20 \%)$. 0 mesmo foi observado nos felinos, com 212 casos de lesões neoplásicas $(45,30 \%), 134$ casos de lesões inflamatórias (28,64\%) e 93 casos de lesões não-neoplásicas (19,87\%).

Quanto à distribuição pelo sexo dos animais acometidos (Quadro 2), as lesões neoplásicas ocorreram tanto em machos quanto em fêmeas, apesar de serem ligeiramente mais frequentes nas fêmeas ( 4.047 casos; $61,61 \%)$. As afecções classificadas como inflamatórias acometeram 1.131 machos $(51,15 \%)$ e 1.080 fêmeas $(48,85 \%)$ e as não-neoplásicas 825 machos $(48,64 \%)$ e 871 fêmeas $(51.36 \%)$. As afecções classificadas como inconclusivas foram observadas em 427 machos (51,14\%) e 408 fêmeas (48,86\%). Não havia informação diagnóstica de 83 machos $(52,87 \%)$ e 74 fêmeas (47.13\%).

Em relação à idade das espécies animais afetadas associada à classificação citológica das afecções (Quadro 3), destaca-se a idade dos caninos e felinos acometidos por lesões neoplásicas. A média de idade dos caninos foi de 119,60 meses, e dos felinos 126,40 meses.

\section{Avaliação dos resultados observados}

Os avanços tecnológicos e o aperfeiçoamento técnico ocorridos nos últimos anos têm propiciado para que o exame citológico seja um método de diagnóstico cada vez mais preciso, com menores taxas de falso negativos ou falso positivos (Ghisleni et al. 2006, Cassali et al. 2007, Simon et al. 2009). Esses eventos, aliados ao fato desse exame facilitar o planejamento de estratégias médicas e cirúrgicas de forma rápida e pouco onerosa (Allen et al.1986, Magalhães et al. 2001, Simeonova et al. 2004, Rossetto et al. 2009, Teixeira et al. 2010), certamente contribuíram para que a citologia fosse considerada uma modalidade de triagem inicial rotineira no diagnóstico e acompanhamento das doenças dos animais no HV da FMVZ-Unesp de Botucatu/SP, durante o período avaliado neste estudo.

Outra possibilidade para o sucesso do exame citológico é a segurança da técnica, pois embora passível de compli-

Quadro 3. Distribuição quanto à idade das espécies animais em relação à classificação citológica das afecções acometendo animais atendidos no Serviço de Citopatologia do Laboratório de Patologia Veterinária, FMVZ-Unesp, Botucatu/SP, de janeiro de 1994 a dezembro de 2008

\begin{tabular}{lccc}
\hline \multirow{2}{*}{ Espécie } & \multicolumn{3}{c}{ Idade média \pm desvio padrão (meses) } \\
\cline { 2 - 4 } & Inflamatórias & Não-neoplásicas & Neoplásicas \\
\hline Avícola & $60,67 \pm 65,92$ & $82,67 \pm 30,02$ & $66,00 \pm 76,21$ \\
Bovina & $50,09 \pm 42,68$ & $42,73 \pm 45,31$ & $95,14 \pm 40,76$ \\
Canina & $83,61 \pm 51,09$ & $92,73 \pm 34,40$ & $119,60 \pm 43,62$ \\
Caprina & $63,33 \pm 36,80$ & $52,00 \pm 18,33$ & $49,00 \pm 1,41$ \\
Equina & $117,69 \pm 58,05$ & $113,80 \pm 73,94$ & $126,55 \pm 58,88$ \\
Felina & $68,47 \pm 41,95$ & $50,27 \pm 36,09$ & $126,40 \pm 34,20$ \\
Leporina & $37,00 \pm 40,50$ & $30,00 \pm 27,49$ & $52,00 \pm 24,98$ \\
Mustela & $36,00 \pm 16,97$ & $24,00 \pm 00,00$ & $00,00 \pm 00,00$ \\
Ovina & $29,14 \pm 15,66$ & $52,80 \pm 27,62$ & $55,00 \pm 21,21$ \\
Primata & Sem informação & $00,00 \pm 00,00$ & Sem informação \\
Roedora & $26,00 \pm 7,48$ & $21,00 \pm 4,24$ & $14,00 \pm 8,49$ \\
Réptil & $24,00 \pm 00,00$ & $00,00 \pm 00,00$ & $00,00 \pm 00,00$ \\
Suína & $00,00 \pm 00,00$ & $00,00 \pm 00,00$ & $00,00 \pm 00,00$
\end{tabular}

cações como sangramento, dor ou mesmo pneumotórax em punções pulmonares (Schmitt 1997), durante os 15 anos de aplicabilidade, não foram encontrados registros de alterações graves que pudessem pôr em risco a vida dos animais submetidos à coleta de amostras para esse exame. Provavelmente isso se deva ao fato de que a conduta citológica a ser adotada pelo Serviço de Citopatologia é decidida em consenso pela equipe veterinária envolvida e procura basear-se em um estudo clínico e laboratorial minucioso, principalmente nos casos de citopunção de estruturas não identificadas pela palpação. Sendo assim, provavelmente o fator complicação da técnica não foi a causa da queda de $2,24 \%$ da solicitação deste exame em relação ao número de atendimentos clínicos no ano de 2005.

Considerando que todas as técnicas de diagnóstico apresentam vantagens e limitações, salienta-se que para obter os benefícios que a citologia proporciona é necessário conhecer as suas indicações e restrições, e que apesar das vantagens apresentadas, o exame citológico não exclui o exame histopatológico, o qual permite detalhamento da arquitetura dos tecidos, o que não pode ser definido pelo exame citológico (Schmitt 1997, Ménard et al. 1986, Magalhães et al. 2001). Ao considerar que os procedimentos de biopsia têm a desvantagem de serem mais invasivos e demorados (Teixeira et al. 2010), a maior valorização da citologia traz uma opção para a chance de um diagnóstico mais precoce (Allen et al. 1986, Magalhães et al. 2001, Simeonova et al. 2004, Rossetto et al. 2009, Teixeira et al. 2010).

Dentre as amostras citológicas de lesões não-inflamatórias neste estudo foram incluídas as alterações não-neoplásicas e as neoplásicas. Neoplasmas possuem critérios nucleares e citoplasmáticos próprios que devem ser distinguidos dos demais processos lesivos que acometem os tecidos (Allen et al. 1986, Raskin \& Meyer 2009). Para tanto, conforme preconizado por Ménard et al. (1986), Larkin (1994), Magalhães et al. (2001), Meyer (2001) e Raskin \& Meyer (2009), amostras colhidas de um mesmo local foram submetidas a duas colorações (Giemsa, que evidencia critérios citoplasmáticos, e Papanicolaou, que evidencia critérios nucleares) (Allen et al. 1986, Raskin \& Meyer 2009). Esse metodo proporcionou boa visualização e interpretação dos componentes celulares e provavelmente contribuiu para a identificação dessas afecções durante o período avaliado.

Neste levantamento, apesar dos 7,28\% dos exames insatisfatórios ou insuficientes serem inferiores aos 16,99\% observados nos levantamentos de Bracarense \& Reis (1997), é relevante destacar que, embora sempre se procure puncionar diversas áreas das lesões para que a possibilidade de obter material de baixa qualidade ou contaminado seja minimizada e que logo após o procedimento de colheita, enquanto o paciente aguarda, as amostras são analisadas para que caso sejam insatisfatórias, uma nova punção possa ser realizada, por se tratar de um hospital escola, a colheita do material certamente nem sempre foi homogênea ou realizada por um mesmo examinador. Esse fato pode ter influenciado nesse percentual, já que a experiência do citopatologista é fundamental na confecção e precisa avaliação das lâminas (Allen et al. 1986, Hellmén \& Lindgren 1989, Raskin \& Meyer 2009). 
Neoplasmas foram prevalentes em cães com faixa etária entre 119,60 $\pm 43,62$ meses e afetaram principalmente fêmeas. Neoplasia é a doença de maior expressão nos cães e, com exceção do histiocitoma, que é mais frequente em cães jovens, a maioria dos processos neoplásicos ocorre em animais mais velhos (Bracarense \& Reis, 1997), provavelmente pela exposição prolongada aos agentes carcinogênicos e à diminuição da resposta imunológica em animais idosos (Priester \& Mckay 1980, Meyer 2001). Quanto ao predomínio de fêmeas, segundo Bracarense \& Reis (1997) e Pires et al. (2003) esse resultado pode ser maior quando no estudo são incluídos os neoplasmas mamários, como no presente trabalho. Neoplasmas mamários têm uma importância significativa na clínica veterinária de pequenos animais, já que podem ser facilmente observados pelo proprietário, avaliados pelo clínico e confirmados pelo exame citológico (Allen et al. 1986, Hellmén \& Lindgren 1989, Cassali et al. 2007, Simon et al. 2009).

\section{CONCLUSÃO}

0 incremento anual observado ao longo dos 15 anos de aplicação da técnica na FMVZ, Unesp-Botucatu/SP, demonstra que o exame citológico é um método de suma importância para confirmar, sugerir ou afastar o diagnóstico de diversas afecções, inclusive neoplasia, em todas as espécies animais.

\section{REFERÊNCIAS}

Allen S.W., Prasse K.W. \& Mahaffey E.A. 1986. Cytologic differentiation of benign from malignant canine mammary tumors. Vet. Pathol. 23:649655.

Bracarense A.P.F.R.L. \& Reis A.C.F. 1997. Citologia aspirativa em animais domésticos: estudo retrospectivo. Semina, Ciênc. Agr. 18:27-32.

Cassali G.D., Gobbi H., Malm C. \& Schimitt F.C. 2007. Evaluation of accuracy of fine needle aspiration cytology for diagnosis of canine mammary tumours: Comparative features with human tumours. Cytopathology 18:191-196.

Ghisleni G., Roccabianca P., Ceruti R., Stefanello D., Bertazzolo W., Bonfanti
U. \& Caniatti M. 2006. Correlation between fine-needle aspiration cytology and histopathology in the evaluation of cutaneous and subcutaneous masses from dogs and cats. Vet. Clin. Pathol. 35:24-30.

Hellmén E. \& Lindgren A. 1989. The accuracy of cytology in diagnosis and DNA analysis of canine mammary tumours. J. Comp. Pathol. 101:443450.

Larkin H.A. 1994. Veterinary cytology: Fine needle aspiration of masses or swellings on animals. Irish Vet. J. 47:65-73.

Magalhães A.M., Ramadinha R.R., Barros C.S.L. \& Peixoto P.V. 2001. Estudo comparativo entre citopatologia e histopatologia no diagnóstico de neoplasmas caninos. Pesq. Vet. Bras. 21:23-32.

Ménard M., Fontaine M. \& Morin M. 1986. Fine needle aspiration biopsy of malignant tumors in dogs and cats: A report of 102 cases. Can. Vet. J. 27:504-510

Meyer D.J. 2001. The essentials of diagnostic cytology in clinical oncology, p.54-62. In: Withrow S.J. \& MacEwen E.G. (Eds), Small Animal Clinical Oncology. $3^{\text {rd }}$ ed. W.B. Saunders, Philadelphia.

Pires M.A., Travassos F.S. \& Pires I. 2003. Dog's neoplasia: A six years descriptive study. Revta Port. Ciênc. Vet. 98:111-118.

Priester W.A. \& McKay F.W. 1980. The occurrence of tumors in domestic animals. Natl Cancer Inst. Monogr. 54:1-210.

Raskin R.E. \& Meyer D.J. 2009. Canine and feline cytology: A color atlas and interpretation guide. $2^{\text {nd }}$ ed. W.B. Saunders, Philadelphia.

Rossetto V.J.V., Moreno K., Grotti C.B., Reis A.C.F. \& Bracarense A.P.F.R.L. 2009. Frequência de neoplasmas em cães diagnosticados por exame citológico: estudo retrospectivo em um hospital-escola. Semina, Ciênc. Agr. 30:189-200.

Schmitt F.C. 1997. Fine-needle aspiration cytology in infectious disease. Revta Soc. Bras. Med. Trop. 30:177-179.

Simeonova G., Simeonov R. \& Krastev S. 2004. Fine-needle aspiration techniques in small animals. Vet. Med. 3/4:60-66.

Simon D., Schoenrock D., Nolte I., Baumgärtner W., Barron R. \& Mischke R. 2009. Cytologic examination of fine-needle aspirates from mammary gland tumors in the dog: diagnostic accuracy with comparison to histopathology and association with postoperative outcome. Vet. Clin. Pathol. 38:521-528.

Teixeira L.V., Lopes S.T.A., Martins D.B., França R.T. \& Fighera R.A. 2010. Punção aspirativa por agulha fina como método de coleta de material para a histopatologia no osteossarcoma canino. Pesq. Vet. Bras. 30:132138. 